\title{
Design and implementation of decision support for tobacco dependence treatment in an inpatient electronic medical record
}

\author{
Steven L Bernstein ${ }^{1,2^{*}}$, June Rosner ${ }^{2}$, Michelle DeWitt ${ }^{2}$, Allen Hsiao², James Dziura ${ }^{2}$, Benjamin Toll ${ }^{2}$ \\ From 7th Annual Conference on the Science of Dissemination and Implementation in Health \\ North Bethesda, MD, USA. 8-9 December 2014
}

\section{Background}

Tobacco dependence treatment for hospitalized smokers results in long-term cessation if treatment continues at least 30 days post-discharge. Methods to leverage inpatient interventions into post-hospitalization care are unclear; health information technology may facilitate ongoing treatment.

\section{Objective}

To develop and test an order set and best practice alert (BPA) addressing tobacco dependence treatment for hospitalized smokers embedded in an electronic medical record (EMR).

\section{Methods}

A 2-arm randomized clinical trial of 250 physicians and patients treated by those physicians. The physician is the unit of randomization. A BPA and order set were developed and embedded in the Epic (Madison, WI) EMR used at 2 hospitals. When an adult patient is admitted to a medical service, a BPA fires if the patient is coded in the EMR as a smoker. For physicians randomized to the intervention, the BPA offers to take the physician to an order set to prescribe tobacco treatment medications and refer the patient to the state smoker's quitline. Additionally, tobacco use disorder" is added to the patient's problem list, and an email is sent to the patient's primary care provider (PCP). In the control arm, a BPA silently fires with no additional actions for the physician.

\section{Results}

Since August 2013, the BPA fired for 4187 patients (1887 intervention, 2300 control). Compared to control arm physicians, intervention physicians were more likely to order tobacco treatment medication $(24 \%$ v. $9 \%$, $\mathrm{P}=0.0001$, Fisher's exact test), populate the problem list with tobacco use disorder ( $46 \%$ v. $4 \%, \mathrm{P}<0.0001)$, and make a referral to the state smokers' quitline $(19 \%$ v. $0 \%$, $\mathrm{P}<0.0001)$. In addition, intervention physicians sent an email to the patient's PCP 1876 (99\%) times.

\section{Conclusion}

Designing and implementing an order set and BPA for tobacco treatment in an EMR is feasible and acceptable. Data on cessation outcomes are pending.

\section{Authors' details \\ ${ }^{1}$ Department of Emergency Medicine, Yale School of Medicine, Yale University, New Haven, CT, 06519, USA. ${ }^{2}$ Yale School of Medicine, Yale-New Haven Hospital, New Haven, CT, 06510, USA.}

Published: 20 August 2015

doi:10.1186/1748-5908-10-S1-A1

Cite this article as: Bernstein et al:: Design and implementation of decision support for tobacco dependence treatment in an inpatient electronic medical record. Implementation Science 2015 10(Suppl 1):A1. 